Mathematical Programming manuscript No.

(will be inserted by the editor)

Pierre Bonami*

Gérard Cornuéjols ${ }^{\star \star}$

Sanjeeb Dash ${ }^{\star \star \star}$

Matteo Fischetti ${ }^{\dagger}$

Andrea Lodi ${ }^{\ddagger}$

\title{
Projected Chvátal-Gomory cuts for Mixed Integer Linear Programs
}

preliminary draft: December 15, 2005

\begin{abstract}
Recent experiments by Fischetti and Lodi show that the first Chvátal closure of a pure Integer Linear Program (ILP) often gives a surprisingly tight approximation of the integer hull. They optimize over the first Chvátal closure by modeling the Chvátal-Gomory (CG) separation problem as a Mixed Integer Linear Program (MILP) which is then solved by a general-purpose MILP solver. Unfortunately, this approach does not extend immediately to the Gomory Mixed Integer (GMI) closure of an MILP, since the GMI separation problem involves the solution of a nonlinear mixed integer program or a parametric MILP. In this paper we introduce a projected version of the CG cuts, and study their practical effectiveness for MILP problems. The idea is to project first the linear programming relaxation of the MILP at hand onto the space of the integer variables, and then to derive Chvátal-Gomory cuts for the projected polyhedron. Though theoretically dominated by GMI cuts, projected CG cuts have the advantage of producing a separation model very similar to the one introduced by Fischetti and Lodi, whose solution can typically be carried out in a reasonable amount of computing time.
\end{abstract}

Key words: mixed integer linear program, Chvátal-Gomory cut, separation problem, projected polyhedron.

\section{Introduction}

Consider first the pure Integer Linear Programming (ILP) problem $\min \left\{c^{T} x\right.$ : $A x \leq b, x \geq 0, x$ integral $\}$ where $A$ is an $m \times n$ rational matrix, $b \in \mathbb{Q}^{m}$, and

Address(es) of author(s) should be given

* Tepper School of Business, Carnegie Mellon University, Pittsburgh PA 15213, USA. Supported in part by a grant from IBM. pbonami@andrew. cmu.edu

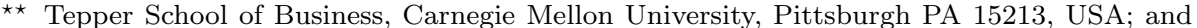
LIF, Faculté des Sciences de Luminy, 13288 Marseille, France. Supported in part by NSF grant DMI-0352885 and ONR grant N00014-03-1-0188. gc0v@andrew. cmu.edu

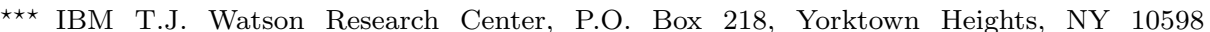
sanjeebd@us.ibm.com

$\dagger$ DEI, University of Padova, via Gradenigo 6A, 35131 Padova, Italy. Supported in part by the EU projects ADONET and ARRIVAL. matteo.fischetti@unipd.it

$\ddagger$ DEIS, University of Bologna, viale Risorgimento 2, 40136 Bologna, Italy Supported in part by the EU projects ADONET and ARRIVAL. alodi@deis.unibo.it 
$c \in \mathbb{Q}^{n}$, along with the two associated polyhedra $P:=\left\{x \in \mathbb{R}_{+}^{n}: A x \leq b\right\}$ and $P_{I}:=\operatorname{conv}\left\{x \in \mathbb{Z}_{+}^{n}: A x \leq b\right\}=\operatorname{conv}\left(P \cap \mathbb{Z}^{n}\right)$.

A Chvátal-Gomory $(C G)$ cut (also known as Gomory fractional cut) [17, 8] is an inequality of the form $\left\lfloor u^{T} A\right\rfloor x \leq\left\lfloor u^{T} b\right\rfloor$ where $u \in \mathbb{R}_{+}^{m}$ is a vector of multipliers, and $\lfloor\cdot\rfloor$ denotes the lower integer part. Chvátal-Gomory cuts are valid inequalities for $P_{I}$. The Chvátal closure of $P$ is defined as

$$
P^{1}:=\left\{x \geq 0: A x \leq b,\left\lfloor u^{T} A\right\rfloor x \leq\left\lfloor u^{T} b\right\rfloor \text { for all } u \in \mathbb{R}_{+}^{m}\right\} .
$$

Thus $P_{I} \subseteq P^{1} \subseteq P$. By the well-known equivalence between optimization and separation [19], optimizing over the first Chvátal closure is equivalent to solving the $C G$ separation problem where we are given a point $x^{*} \in \mathbb{R}^{n}$ and asked to find a hyperplane separating $x^{*}$ from $P^{1}$ (if any). Without loss of generality we can assume that $x^{*} \in P$, since all other points can be cut by simply enumerating the members of the original inequality system $A x \leq b, x \geq 0$. Therefore, the separation problem we are actually interested in reads:

CG-SEP: Given any point $x^{*} \in P$ find (if any) a CG cut that is violated by $x^{*}$, i.e., find $u \in \mathbb{R}_{+}^{m}$ such that $\left\lfloor u^{T} A\right\rfloor x^{*}>\left\lfloor u^{T} b\right\rfloor$, or prove that no such $u$ exists.

It was proved by Eisenbrand [15] that CG-SEP is NP-hard, so optimizing over $P^{1}$ also is. Fischetti and Lodi [16] recently addressed the issue of evaluating the practical strength of $P^{1}$ in approximating $P_{I}$. Their approach is to model the CG separation problem as an MILP, which is then solved through a generalpurpose MILP solver. To be more specific, given an input point $x^{*} \in P$ to be separated, CG-SEP calls for a CG cut $\alpha^{T} x \leq \alpha_{0}$ which is (maximally) violated by $x^{*}$, where $\alpha=\left\lfloor u^{T} A\right\rfloor$ and $\alpha_{0}=\left\lfloor u^{T} b\right\rfloor$ for some $u \in \mathbb{R}_{+}^{m}$. Hence, if $A_{j}$ denotes the $j$ th column of $A$, CG-SEP can be modeled as:

$$
\begin{array}{ll}
\max & \alpha^{T} x^{*}-\alpha_{0} \\
& \alpha_{j} \leq u^{T} A_{j}, \quad \text { for } j=1, \ldots, n \\
& \alpha_{0}+1-\epsilon \geq u^{T} b, \\
& u_{i} \geq 0, \quad \text { for } i=1, \ldots, m \\
& \alpha_{j} \text { integer, } \quad \text { for } j=0, \ldots, n
\end{array}
$$

where $\epsilon$ is a small positive value. In the model above, the integer variables $\alpha_{j}$ $(j=1, \ldots, n)$ and $\alpha_{0}$ play the role of coefficients $\left\lfloor u^{T} A_{j}\right\rfloor$ and $\left\lfloor u^{T} b\right\rfloor$ in the CG cut, respectively. Hence the objective function (2) gives the amount of violation of the CG cut evaluated for $x=x^{*}$, that we want to maximize. Because of the sign of the objective function coefficients, the rounding conditions $\alpha_{j}=\left\lfloor u^{T} A_{j}\right\rfloor$ can be imposed through upper bound conditions on variables $\alpha_{j}(j=1, \ldots, n)$, as in (3), and with a lower bound condition on $\alpha_{0}$, as in (4). Note that this latter constraint requires the introduction of a small value $\epsilon$ so as to avoid an integer $u^{T} b$ be rounded to $u^{T} b-1$.

Model (2)-(6) can also be explained by observing that $\alpha^{T} x \leq \alpha_{0}$ is a CG cut if and only if $\left(\alpha, \alpha_{0}\right)$ is an integral vector, as stated in (6), and $\alpha^{T} x \leq$ 
$\alpha_{0}+1-\epsilon$ is a valid inequality for $P$, as stated in (3)-(5) by using the well-known characterization of valid inequalities for a polyhedron due to Farkas.

Unfortunately, model (2)-(6) does not extend immediately to the mixed integer case, where one typically concentrates on the stronger Gomory Mixed Integer (GMI) cuts [18]. Although it is easy to find a GMI cut that separates a basic solution of the linear programming relaxation that is not integer feasible, separating other points by GMI cuts is NP-hard [7], [13]. One can define the Gomory mixed integer closure in an analogous way to the Chvátal closure: add all the GMI cuts to the original formulation. Not only is the separation problem for the Gomory mixed integer closure NP-hard, but there is no MILP model like (2)-(6) known for it. Indeed, one faces the solution of a nonlinear [14] or parametric [4] mixed integer problem for the separation of GMI cuts. In this paper we introduce a projected version of the classical CG cuts, and study their strength on general instances and on some specific classes of MILP problems. The idea is to project first the linear programming relaxation of the MILP at hand onto the space of the integer variables, and then to derive Chvátal-Gomory cuts for the projected polyhedron. Though theoretically dominated by GMI cuts, projected CG cuts have the advantage of producing an MILP separation model very similar to (2)(6), hence its solution can typically be carried out in a reasonable amount of computing time. Also, it can be conjectured that projected CG cuts are more "combinatorial" in nature than GMI cuts, and can be quite effective for a large class of MILPs - notably, those where the continuous variables are only used to model some feasibility condition, possibly by using big-M coefficients, and are not present in the objective function, as, e.g., those addressed in [9].

The present paper is organized as follows. In Section 2 we define more precisely our projected CG cuts, give a MILP formulation of the associated separation problem and describe their relation to GMI cuts. In Section 3, we prove a theorem showing that projected CG cuts are equivalent to split cuts [11] in which one term of the disjunction has an empty intersection with the original formulation. In Section 4 we address the important issue of whether projected CG cuts are likely to be effective, at least for some classes of problems. Computational results on all the mixed MILP instances taken from the MIPLIB 3.0 library [5] are presented in Section 5, as well as on instances of the asymmetric traveling salesman with time windows. These results show the effectiveness of projected CG cuts both on general instances and on instances arising in specific contexts. Concluding remarks and future directions of research are addressed in Section 6.

\section{Projected Chvátal-Gomory cuts}

The computational results reported in [16] show that $P^{1}$ often gives a surprisingly tight approximation of $P$, so a natural question is whether the same result generalizes to mixed integer linear programming problems. 

form

In this paper, we consider a Mixed Integer Linear Program (MILP) of the

$$
\min \left\{c^{T} x+f^{T} y: A x+C y \leq b, x \geq 0, x \text { integral, } y \geq 0\right\}
$$

where $A$ and $C$ are $m \times n$ and $m \times r$ rational matrices respectively, $b \in \mathbb{Q}^{m}$, $c \in \mathbb{Q}^{n}$, and $f \in \mathbb{Q}^{r}$. We also consider the two following polyhedra in the $(x, y)$ space:

$$
\begin{aligned}
& P(x, y):=\left\{(x, y) \in \mathbb{R}_{+}^{n} \times \mathbb{R}_{+}^{r}: A x+C y \leq b\right\} \\
& P_{I}(x, y):=\operatorname{conv}(\{(x, y) \in P(x, y): x \text { integral }\}) .
\end{aligned}
$$

Our first order of business is to extend the classical definition of Chvátal-Gomory cuts to the mixed integer case, in such a way that the resulting separation problem retains as much as possible the simple structure of model (2)-(6). To this end, we define the projection of $P(x, y)$ onto the space of the $x$ variables as:

$$
\begin{aligned}
P(x) & :=\left\{x \in \mathbb{R}_{+}^{n}: \text { there exists } y \in \mathbb{R}_{+}^{r} \text { s.t. } A x+C y \leq b\right\} \\
& =\left\{x \in \mathbb{R}_{+}^{n}: u^{k} A \leq u^{k} b, k=1, \ldots, K\right\} \\
& =:\left\{x \in \mathbb{R}_{+}^{n}: \bar{A} x \leq \bar{b}\right\}
\end{aligned}
$$

where $u^{1}, \ldots, u^{K}$ are the (finitely many) extreme rays of the projection cone $\left\{u \in \mathbb{R}_{+}^{m}: u^{T} C \geq 0^{T}\right\}$. Note that the rows of the linear system $\bar{A} x \leq \bar{b}$ are of Chvátal rank 0 with respect to $P(x, y)$, i.e, no rounding argument is needed to prove their validity.

We then define a projected Chvátal-Gomory (pro-CG) cut as a CG cut derived from the system $\bar{A} x \leq \bar{b}, x \geq 0$, i.e., an inequality of the form $\left\lfloor w^{T} \bar{A}\right\rfloor x \leq\left\lfloor w^{T} \bar{b}\right\rfloor$ for some $w \geq 0$. Since any row of $\bar{A} x \leq \bar{b}$ can be obtained as a linear combination of the rows of $A x \leq b$ with multipliers $\bar{u} \geq 0$ such that $\bar{u}^{T} C \geq 0^{T}$, it follows that a pro-CG cut can equivalently (and more directly) be defined as an inequality of the form

$$
\left\lfloor u^{T} A\right\rfloor x \leq\left\lfloor u^{T} b\right\rfloor \quad \text { for any } u \geq 0 \text { such that } u^{T} C \geq 0^{T} .
$$

As such, its associated separation problem can be modeled as a simple extension of (2)-(6), through the following MILP:

$$
\begin{array}{ll}
\max & \alpha^{T} x^{*}-\alpha_{0} \\
& \alpha_{j} \leq u^{T} A_{j}, \quad \text { for } j=1, \ldots, n \\
& 0 \leq u^{T} C_{j}, \quad \text { for } j=1, \ldots, r \\
& \alpha_{0}+1-\epsilon \geq u^{T} b \\
& u_{i} \geq 0, \quad \text { for } i=1, \ldots, m \\
& \alpha_{j} \quad \text { integer, } \quad \text { for } j=0, \ldots, n .
\end{array}
$$




\section{Connection with split cuts}

In this section, we relate the projected Chvátal-Gomory cuts to known cuts for MILP. For this, it will be convenient to define the Chvátal-Gomory closure of $P(x, y)$ as the intersection of $P(x, y)$ with all the pro-CG cuts (viewed as inequalities $\alpha^{T} x+0^{T} y \leq \alpha_{0}$ in $\mathbb{R}^{n} \times \mathbb{R}^{r}$ ). We denote the Chvátal-Gomory closure of $P(x, y)$ by $P^{1}(x, y)$. Since the intersection of all pro-CG cuts is a polyhedron, it follows that $P^{1}(x, y)$ also is.

Split cuts were introduced by Cook, Kannan and Schrijver [11]. They are obtained as follows. For any $\pi \in \mathbb{Z}^{n}$ and $\pi_{0} \in \mathbb{Z}$, the disjunction $\pi^{T} x \leq \pi_{0}$ or $\pi^{T} x \geq \pi_{0}+1$ is valid for MILP. In other words, $P_{I}(x, y) \subseteq \operatorname{conv}\left(\Pi_{0} \cup \Pi_{1}\right)$ where

$$
\begin{aligned}
& \Pi_{0}:=P(x, y) \cap\left\{(x, y): \pi^{T} x \leq \pi_{0}\right\} \\
& \Pi_{1}:=P(x, y) \cap\left\{(x, y): \pi^{T} x \geq \pi_{0}+1\right\} .
\end{aligned}
$$

A valid inequality for $\operatorname{conv}\left(\Pi_{0} \cup \Pi_{1}\right)$ is called a split cut. The convex set obtained by intersecting $P(x, y)$ with all the split cuts is called the split closure of $P(x, y)$. Cook, Kannan and Schrijver proved that the split closure of $P(x, y)$ is a polyhedron. Nemhauser and Wolsey [24] proved that the split closure and the Gomory mixed integer closure are identical sets. See [12] for a direct proof of this result. Projected Chvátal-Gomory cuts are dominated by GMI cuts, and therefore $P^{1}(x, y)$ contains the split closure of $P(x, y)$. The following result gives the precise relation between the two classes of cuts.

Theorem 1. Let $S(x, y)$ denote the intersection of $P(x, y)$ with all the split cuts where one of the sets $\Pi_{0}, \Pi_{1}$ defined in (20) and (21) is empty. Then

$$
P^{1}(x, y)=S(x, y) \text {. }
$$

Proof. First we prove $S(x, y) \subseteq P^{1}(x, y)$. Consider an inequality that defines a facet of $P^{1}(x, y)$. If it is valid for $P(x, y)$, then it is clearly valid for $S(x, y)$. So we may assume that the facet of $P^{1}(x, y)$ is defined by a pro CG cut $\pi^{T} x \leq \pi_{0}$. By the Chvátal-Gomory procedure $\pi^{T} x \leq \beta$ must be a valid inequality for $P(x, y)$ for some $\beta<\pi_{0}+1$. This implies that $\Pi_{1}:=P(x, y) \cap\left\{(x, y): \pi^{T} x \geq \pi_{0}+1\right\}$ is empty. Therefore $\operatorname{conv}\left(\Pi_{0} \cup \Pi_{1}\right)=\Pi_{0}$. This implies that $\pi^{T} x \leq \pi_{0}$ is valid for $\operatorname{conv}\left(\Pi_{0} \cup \Pi_{1}\right)$, proving that it is a split cut. Furthermore this split cut is valid for $S(x, y)$ since $\Pi_{1}=\emptyset$.

Conversely, we prove $P^{1}(x, y) \subseteq S(x, y)$. Consider a valid inequality for $S(x, y)$. If it is valid for $P(x, y)$, then it is clearly valid for $P^{1}(x, y)$. So we only need to consider a valid inequality for $S(x, y)$ that arises from a split cut where one of the sets $\Pi_{0}, \Pi_{1}$ is empty, for some $\pi \in \mathbb{Z}^{n}$ and $\pi_{0} \in \mathbb{Z}$. Without loss of generality we may assume that $\Pi_{1}=\emptyset$. In other words, the inequality under consideration is valid for $\Pi_{0}$. We will show that $P^{1}(x, y) \subseteq \Pi_{0}$. Since all the inequalities that define $\Pi_{0}$ are valid for $P(x, y)$ except possibly for the inequality $\pi^{T} x \leq \pi_{0}$, it suffices to show that $\pi^{T} x \leq \pi_{0}$ is a pro-CG cut. Let

$$
\beta=\max \begin{aligned}
& \pi^{T} x \\
& x \in P(x, y) .
\end{aligned}
$$


Since $\Pi_{1}=\emptyset$, it follows that $\beta<\pi_{0}+1$. Therefore $\pi^{T} x \leq \beta$ is a valid inequality for $P(x, y)$. Since $y$ does not appear in this inequality, it is also valid for $P(x)$. The Chvátal-Gomory procedure implies that $\pi^{T} x \leq\lfloor\beta\rfloor \leq \pi_{0}$ is a pro-CG cut.

\section{On the strength of projected CG cuts}

In this section we address the important practical issue of the expected strength of the projected CG cuts. For this it is useful to distinguish between two extreme cases of MILPs: those where the essence of the problem is in the optimization of the integer variables $x$, and those where optimizing over the continuous variables is the key. This can be illustrated by the following simple example in two variables $x$ and $y$ (with $x$ integer and $y$ continuous): $P(x, y)$ is the polytope defined by the inequalities $x+y \leq 3 / 2, y \leq x$ and $x, y \geq 0$. Observe that the pro-CG cut $x \leq 1$ cuts off the vertex $(3 / 2,0)$, but there is no pro-CG cut which cuts off the non-integral vertex $(3 / 4,3 / 4)$. Thus, if the objective is to maximize $x$, pro-CG cuts help, and optimizing over $P^{1}(x)=P_{I}(x)$ yields the optimal solution. On the other hand, if the objective is to maximize $y$, pro-CG cuts do not help. More generally, suppose that the projection of the optimum of the MILP relaxation $P(x, y)$ belongs to the first Chvátal closure $P^{1}(x)$. In this case, no pro-CGcut can cut off that point, although there might possibly be a huge gap between the MILP and its relaxation.

On the other hand, pro-CG cuts are well suited to handle those MILPs where the continuous variables are only used to model some feasibility condition, possibly by using big-M coefficients, but are not present in the objective function. Indeed, take any inequality of the form $g^{T} x+0^{T} y \leq g_{0}$ that is valid for $P_{I}(x, y)$. In particular, if $f=0$ and $z^{*}$ denotes the optimum objective value of MILP, the inequality $c^{T} x+f^{T} y \geq z^{*}$ is such a valid inequality for $P_{I}(x, y)$. Then $g^{T} x \leq g_{0}$ must also be valid for the projected integer polyhedron $P_{I}(x)$, hence it is of finite Chvátal rank, say $q$, with respect to system $\bar{A} x \leq \bar{b}, x \geq 0$. This implies that $g^{T} x \leq g_{0}$ is indeed a pro-CG cut (of the same rank $q$ ) with respect to the original system $A x+C y \leq b,(x, y) \geq 0$. As a consequence, MILPs where the continuous variables do not appear in the objective function can always be optimized to proven optimality by only using (of course in an iterative way) pro-CG cuts.

A class of problems where (even rank 1) pro-CG cuts are likely to be really effective has been recently addressed by Codato and Fischetti [9]. These authors considered a basic 0-1 ILP of the form

$$
\min \left\{c^{T} x: F x \leq g, x \in\{0,1\}^{n}\right\}
$$

amended by a set of "conditional" linear constraints involving additional continuous variables $y$, of the form

$$
x_{i}=1 \Rightarrow w_{i}^{T} y \leq w_{i 0}, \text { for all } i \in I
$$

plus a (possibly empty) set of $k$ (say) "unconditional" constraints on the continuous variables $y$, namely

$$
D y \leq d .
$$


Note that the continuous variables $y$ do not appear in the objective functionthey are only introduced to force some feasibility properties of the $x$ 's. A familiar example of a problem of this type is the classical Asymmetric Traveling Salesman Problem (ATSP) with time windows, called TW-ATSP in the sequel. Here the binary variables $x_{i j}$ are the usual arc variables, and the continuous variables $y_{i}$ give the arrival time at city $i$. Each arc $(i, j)$ has duration $d_{i j}$, and each city $i$ has to be visited within the time window $\left[e_{i}, l_{i}\right]$. For this problem, the basic formulation (22) contains the standard ATSP out- and in-degree equations (plus any other ATSP constraints such as subtour elimination etc.). Implications (23) are of the form

$$
x_{i j}=1 \Rightarrow y_{j} \geq y_{i}+d_{i j}
$$

whereas (24) bounds the arrival time at city $i$

$$
e_{i} \leq y_{i} \leq l_{i} \text { for all } i \in I \text {. }
$$

Another example is the map labeling problem [21], where the binary variables are associated with the relative position of two labels to be placed on a map, the continuous variables give their placement coordinates, and the conditional constraints impose non-overlapping conditions of the type "if label $i$ is placed on the right of label $j$, then the placement coordinates of $i$ and $j$ must obey a certain linear inequality giving a suitable separation condition".

The usual way implications (23) are modeled within the MILP framework is to use the famous big- $M$ method, where large positive coefficients $M$ are introduced to activate/deactivate the conditional constraints to be added to the basic model (22), as in:

$$
w_{i}^{T} y+M\left(x_{i}-1\right) \leq w_{i 0} \text { for all } i \in I .
$$

For example, the TW-ATSP implications (25) are usually modeled as:

$$
y_{i}-y_{j}+M x_{i j} \leq M-d_{i j} .
$$

It is known however that, due to the presence of the big-M coefficients, the LP relaxation of the resulting MILP model is typically very poor. As a matter of fact, the $x$ solutions of the LP relaxation are only marginally affected by the addition of the $y$ variables and of the associated constraints. To remedy this behavior, Codato and Fischetti proposed the use of the so-called Combinatorial Benders' (CB) cuts:

$$
\sum_{i \in Q} x_{i} \leq|Q|-1
$$

where $Q \subseteq I$ induces a minimal (irreducible) infeasible subsystem of (23)-(24), i.e., an inclusion-minimal set of row-indices of system (23) such that the linear subsystem

$$
\begin{aligned}
& w_{i}^{T} y \leq w_{i 0}, \text { for all } i \in Q \\
& D y \leq d
\end{aligned}
$$


has no feasible (continuous) solution $y$. In a sense, CB cuts try to project in a purely combinatorial way the feasibility requirement in the $x$ space (hence their name). They can be viewed as an attempt to distill automatically some combinatorial information from the input MILP model. In this process, the role of the big-M terms in the MILP model vanishes - only implications (23) are relevant, no matter how they are modeled. The computational results reported in [9] show that CB cuts can be really effective for specific classes of MILPs that are notoriously very hard to solve: even with a simple implementation of the $\mathrm{CB}$ cut separation procedure, the use of $\mathrm{CB}$ cuts results in a speed-up by several orders of magnitude compared to the best commercial MILP solvers on some important classes of MILPs.

The next proposition shows that $\mathrm{CB}$ cuts are a special case of projected $\mathrm{CG}$ cuts.

Theorem 2. Combinatorial Benders cuts are projected CG cuts.

Proof. Consider a combinatorial Benders cut $\sum_{i \in Q} x_{i} \leq|Q|-1$ where $Q$ induces a minimal infeasible system of (23)-(24). Maximizing $\sum_{i \in Q} x_{i}$ over the feasible region $P(x, y)$ of the big-M MILP yields an objective value $\beta<|Q|$, since all $x_{i}$ cannot be 1 . Therefore the Chvátal-Gomory procedure implies that $\sum_{i \in Q} x_{i} \leq$ $|Q|-1$ is a CG cut for $P(x, y)$. Since the $y$ variables do not appear in $\sum_{i \in Q} x_{i} \leq$ $|Q|-1$, it is also a projected CG cut.

Projected CG cuts can however be much stronger than CB cuts, in that they can exploit all the information contained in the basic model (22). We illustrate this through the TW-ATSP example. Suppose you have a simple dipath $P$ of cardinality $k$ (say) from a certain node $a$ to a certain node $b$, whose total duration exceeds the difference $l_{b}-e_{a}$. To fix the ideas, let the dipath be $P:=\{(0,1),(1,2),(2,3),(3,4)\}$, hence $k=4$, and let $d_{i j}=10$ for all $(i, j) \in P$, with $e_{0}=5$ and $l_{4}=40$. The TW-ATSP model includes the following constraints (we choose $M=100$ ), plus the nonnegativity constraints on the $x$ variables:

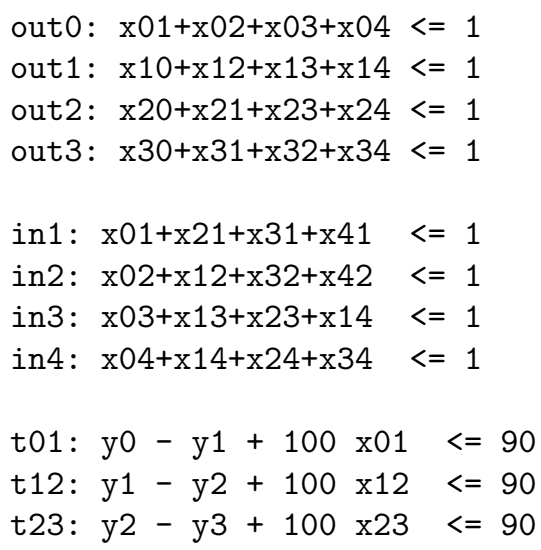


t34: $\mathrm{y}^{3}-\mathrm{y} 4+100 \mathrm{x} 34<=90$

early0: $-\mathrm{y} 0<=-5$

late $4: \quad$ y $4<=40$

Clearly, every feasible TW-ATSP solution has to satisfy the infeasible path constraint $x(P):=\sum_{(i, j) \in P} x_{i j} \leq|P|-1$, i.e., $x_{01}+x_{12}+x_{23}+x_{34} \leq 3$ in our case. This cut is a $\mathrm{CB}$ cut, since clearly $P$ induces an infeasible subset of system (25)-(26). Because of the discussion above, the cut is also a projected CG cut. This can easily be verified by maximizing the left-hand-side of the cut (namely, $\mathrm{x} 01+\mathrm{x} 12+\mathrm{x} 23+\mathrm{x} 34$ ) over the above system of linear constraints, obtaining an optimal value of 3.95 (to be rounded down to 3 ). However, the path infeasibility constraint is rather weak in that it does not take into account the presence of the out- and in-degree constraints, as in the stronger tournament inequality $x([P]) \leq|P|-1$ proposed by Ascheuer, Fischetti and Grötschel [2], where $P$ is any infeasible path, and $[P]:=\{(i, j)$ : node $i$ precedes node $j$ in $P\}$ is its transitive closure. In our example, the tournament inequality reads $\mathrm{x} 01+\mathrm{x} 02+\mathrm{x} 03+\mathrm{x} 04+\mathrm{x} 12+\mathrm{x} 13+\mathrm{x} 14+\mathrm{x} 23+\mathrm{x} 24+\mathrm{x} 34<=3$. Optimizing the left-andside over the LP system above produces an optimal solution value of 3.9875 (still rounded down to 3 ) showing that the tournament inequality is a projected CG cut.

\section{Computational results}

In this section we report the outcome of our experiments on a test-bed made up of 43 mixed-integer problems from MIPLIB 3.0 [5]. The approach follows the scheme used in [16], i.e., we implemented a pure cutting plane algorithm where, at each iteration, pro-CG cuts are separated by solving the separation problem (14)-(19) through a standard MILP solver. In order to speedup the overall computation, the MILP solver is aborted whenever its incumbent solution does not improve for a certain number of branching nodes. Our implementation of the cutting-plane method uses the commercial software ILOG-Cplex 9.0 as the LP solver, whereas the separation problem is solved through ILOG-Cplex 9.0 MILP solver with "mip emphasis 4" parameter; see [20]. All computing times refer to a $3.2 \mathrm{Ghz}$ Pentium $4 \mathrm{PC}$ with $2 \mathrm{~GB}$ of RAM.

In particular, Table 1 reports the results for the cutting plane algorithm using pro-CG cuts while Tables 2-3 compare those results with other general-purpose cuts.

Table 1 is partitioned into three parts: at the top we report 10 instances for which we have been able to optimize over the Chvátal-Gomory closure in the time limit of $20 \mathrm{CPU}$ minutes (1,200 CPU seconds), then we have 26 instances in which our cutting plane procedure exceeded such a time limit, and finally, we report 7 instances for which the algorithm did not find any cut and proved that none exists. For each instance, we report besides its name (instance), the numbers of 


\begin{tabular}{|c|c|c|c|c|c|c|c|c|}
\hline \multirow[b]{2}{*}{ instance } & \multirow[b]{2}{*}{$n$} & \multirow[b]{2}{*}{$r$} & \multirow[b]{2}{*}{$r_{c}$} & \multicolumn{5}{|c|}{ pro-CG } \\
\hline & & & & \# iter & \# cuts & $\begin{array}{l}\text { CPU } \\
\text { time }\end{array}$ & & $\begin{array}{l}\text { \% gap } \\
\text { closed }\end{array}$ \\
\hline bell3a & 71 & 62 & 46 & 70 & 241 & 65.3 & & 48.10 \\
\hline bell5 & 58 & 46 & 32 & 36 & 126 & 4.4 & & 91.73 \\
\hline $\mathrm{g}$ & 55 & 86 & 55 & 35 & 168 & 6.8 & & 81.77 \\
\hline fixn & 378 & 500 & 416 & 34 & 83 & 42.9 & & 67.51 \\
\hline $\mathrm{khl}$ & 4 & 1,326 & 1,249 & 5 & 13 & 3.5 & & 4.70 \\
\hline nos & 100 & 28 & 0 & 39 & 118 & 68.0 & & \\
\hline $\mathrm{re}$ & 55 & 9,502 & 177 & 7 & 15 & 5.1 & & 0.00 \\
\hline se & 240 & 472 & 232 & 29 & 89 & 34.2 & & 51.41 \\
\hline vpn & & 210 & 0 & 27 & 53 & 14.9 & & 100.00 \\
\hline vpn & 20 & 210 & 0 & 89 & 275 & $1,021.9$ & & 62.86 \\
\hline $10 \mathrm{t}$ & $\overline{0}$ & 225 & 225 & 455 & 2,001 & $1,200.0$ & $\geq$ & 57.14 \\
\hline & & 850 & 1 & 62 & 215 & $1,200.0$ & $\bar{z}$ & 28.04 \\
\hline & & 89 & 0 & 363 & 1,032 & $1,200.0$ & $\geq$ & 36.40 \\
\hline & 552 & 3,321 & 1 & 1 & 0 & $1,200.0$ & $\sum$ & 0.00 \\
\hline & 6 & 465 & 1 & 4 & 3 & $1,200.0$ & $\geq$ & 0.01 \\
\hline & 75 & 3 & 73 & 46 & 132 & $1,200.0$ & $\geq$ & 47.25 \\
\hline & 192 & 694 & ,068 & 186 & 433 & $1,200.0$ & & \\
\hline & 1,254 & 44 & 0 & 89 & 1,556 & $1,200.0$ & $\geq$ & 4.83 \\
\hline & & 7 & 7 & 3 & 2 & $1,200.0$ & $\geq$ & 19.19 \\
\hline & & 720 & 432 & 171 & 427 & $1,200.0$ & $\geq$ & 86.60 \\
\hline & & 816 & $c \Omega$ & 383 & 1,660 & $1,200.0$ & $\geq$ & 94.84 \\
\hline & & 504 & & 76 & 306 & $1,200.0$ & $\geq$ & 94.93 \\
\hline & & 768 & & 138 & 381 & $1,200.0$ & $\geq$ & 58.96 \\
\hline & & 480 & 264 & 49 & 93 & $1,200.0$ & $\bar{z}$ & 64.53 \\
\hline & & 12 & 12 & 345 & 686 & $1,200.0$ & $\sum$ & 0.00 \\
\hline & 60 & 14 & 14 & 111 & 720 & $1,200.0$ & $\bar{z}$ & 0.00 \\
\hline & 5,323 & 2 & 0 & 87 & 267 & $1,200.0$ & $\geq$ & 1.27 \\
\hline & & 1 & 1 & 303 & 852 & $1,200.0$ & $\bar{z}$ & 34.92 \\
\hline & 25 & 1 & & 31 & 889 & $1,200.0$ & $\geq$ & 3.86 \\
\hline & & 6 & & 7 & 8 & $1,200.0$ & $\overline{>}$ & 4.32 \\
\hline $\mathrm{p}$ & 64 & & & 4 & 5 & $1,200.0$ & $z$ & 0.68 \\
\hline & 48 & & & 7 & 8 & 1,2 & $z$ & 10.71 \\
\hline & 417 & 124 & & 14 & 715 & $1,200.0$ & $\geq$ & 7.32 \\
\hline & 1,417 & 124 & 124 & 18 & 1,340 & $1,200.0$ & $\sum$ & 8.61 \\
\hline & & 241 & 1 & & 1,715 & $1,200.0$ & 5 & 0.03 \\
\hline & 6, & 81 & 1 & 354 & 1,222 & $1,200.0$ & & 7.68 \\
\hline mas & 150 & 1 & 1 & 1 & 0 & 0.0 & & 0.00 \\
\hline & & 1 & & & 0 & 0.0 & & 0.00 \\
\hline & & 96 & & & 0 & 0.0 & & 0.00 \\
\hline 11 & 96 & 10,862 & 7,489 & 1 & 0 & 0.4 & & 0.00 \\
\hline modg & 98 & 324 & 324 & 1 & 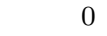 & 0.0 & & 0.00 \\
\hline $\mathrm{pk}$ & & 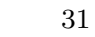 & & 1 & - & 0.0 & & 0.00 \\
\hline rgn & 100 & 80 & 80 & 1 & 0 & 0.6 & & 0.00 \\
\hline
\end{tabular}

Table 1. MILPs of the MIPLIB 3.0. Note that for instances dsbmip and noswot there is no gap between the initial LP (though fractional) solution and the optimal value, while for the optimal solution of instance arki001 we used the best known of value 7,579,599.8078

integer $(n)$ and continuous variables $(r)$ and the number of continuous variables with a nonzero coefficient in the objective function $\left(r_{c}\right)$. Then, we report for the pro-CG cuts, the number of iterations (\# iter) and the number of separated cuts (\# cuts), the CPU time and the percentage of gap closed (\% gap closed) computed as $100 \frac{\text { opt_value }\left(P^{1}\right)-\text { opt_value }(P)}{\text { opt_value }\left(P_{I}\right)-\text { opt_value }(P)}$. 
The results in Table 1 show that the projected Chvátal-Gomory closure can be an effective approximation of the integer hull of MILPs. The average gap closed over 41 instances $^{1}$ is around $29 \%$. On the other hand, as expected, there are several (at least 7) instances for which no pro-CG cut exists. For 11 instances out of 41 , optimizing over the projected Chvátal-Gomory closure (up to the time limit of 1200 seconds) produced absolutely no improvement. For the 30 remaining instances, however, the average gap closed is around $40 \%$. On certain instances (bel15, gesa2), the projected Chvátal-Gomory closure closes over $90 \%$ of the gap. On vpm1 the projected Chvátal-Gomory closure even closes $100 \%$ of the gap. This is impressive considering that the pro-CG cuts are also attractive from a numerical point of view: they tend to deteriorate less rapidly than the GMI cuts read from the LP tableau.

In Tables 2 and 3 we report comparisons with classical families of cutting planes that are valid for the Gomory mixed integer closure: Gomory Mixed Integer cuts from the optimal tableau of the LP relaxation, MIR cuts (Marchand and Wolsey [22]) and lift-and-project cuts [3]. Specifically, the columns GMI and MIR in Table 2 refer to one round of Gomory Mixed Integer cuts, and of Mixed Integer Rounding cuts respectively, as implemented in the COIN-OR cut generator [10]. The column L\&P in Table 3 refers to the gap closed by the liftand-project closure plus a strengthening step, as implemented by Bonami and Minoux [6]. Note that we set a time limit of $20 \mathrm{CPU}$ minutes on each run: 5 instances were interrupted because of the time limit. Tables 2 and 3 show the improvement achieved by the projected Chvátal-Gomory closure when it is applied subsequently to the three other families of cuts, either separately, or all together (the column GMI+MIR+L\&P was obtained by applying first the GMI and MIR cuts and then, starting from the resulting solution, the L\&P separation step). An additional time limit of $20 \mathrm{CPU}$ minutes was set on generating projected Chvátal-Gomory cuts, for all the runs. Note that for instances where we only partially optimize over the projected Chvátal-Gomory closure, it can happen that the pro-CG gap closed is better than the GMI+pro-CG gap closed (blend2 is such an instance). We can make the following observations. The pro-CG cuts can sometimes be vastly superior to the other families of cuts (be115, gesa2, vpm1). The average gap closed by the projected Chvátal-Gomory closure (29\%) is comparable to that closed by GMI cuts (24\%), MIR cuts (23\%) and the lift-and-project closure (35\%). Tables 2 and 3 show that pro-CG cuts are quite different from the other families of cuts. Adding the pro-CG cuts to the GMI cuts improves the average closed gap from $24 \%$ to $41 \%$. Adding them to MIR cuts improves it from $23 \%$ to $40 \%$, and adding them to the lift-and-project closure improves it from $35 \%$ to $49 \%$. Finally, adding the pro-CG cuts to all the other cuts combined still improves the average gap from $48 \%$ to $55 \%$. The case of egout is interesting: the gap is closed completely by combining the 4 types of cuts but not without the pro-CG cuts. Other interesting cases are bell3a and

1 Instances dsbmip and noswot are not considered in the average. 


\begin{tabular}{|c|c|c|c|c|c|c|}
\hline \multirow[b]{2}{*}{ instance } & \multicolumn{6}{|c|}{$\%$ gap closed } \\
\hline & GMI & & $\begin{array}{c}\text { GMI + } \\
\text { pro-CG }\end{array}$ & MIR & & $\begin{array}{c}\text { MIR + } \\
\text { pro-CG }\end{array}$ \\
\hline bell3a & 45.10 & & 78.71 & 19.06 & & 60.94 \\
\hline bell5 & 14.53 & & 92.64 & 0.40 & & 91.88 \\
\hline egout & 40.26 & & 84.18 & 57.14 & & 92.74 \\
\hline fixnet6 & 10.27 & & 75.96 & 69.92 & & 82.48 \\
\hline khb05250 & 74.91 & & 74.91 & 77.92 & & 77.92 \\
\hline noswot & - & & - & - & & 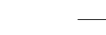 \\
\hline rentacar & 0.00 & & 0.00 & 0.00 & & 0.00 \\
\hline set1ch & 38.11 & & 70.41 & 38.27 & & 69.41 \\
\hline vpm1 & 10.00 & & 100.00 & 33.08 & & 100.00 \\
\hline vpm2 & 13.00 & & 64.70 & 31.52 & & 68.55 \\
\hline 10teams & 100.00 & & 100.00 & 0.00 & $\geq$ & 57.14 \\
\hline arki001 & 34.72 & $\geq$ & 36.01 & 7.03 & $\bar{z}$ & 33.19 \\
\hline blend2 & 16.29 & $\bar{z}$ & 31.39 & 14.39 & $\bar{z}$ & 32.06 \\
\hline dano3mip & 0.01 & $\geq$ & 0.01 & 0.01 & $\geq$ & 0.01 \\
\hline danoint & 0.22 & $\bar{z}$ & 0.22 & 0.49 & $\bar{z}$ & 0.49 \\
\hline dcmulti & 47.25 & $\bar{z}$ & 67.88 & 7.49 & $\bar{z}$ & 54.23 \\
\hline dsbmip & - & & - & - & & 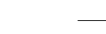 \\
\hline fiber & 72.18 & $\geq$ & 75.53 & 25.27 & $\geq$ & 30.12 \\
\hline flugpl & 11.74 & $\bar{z}$ & 11.74 & 0.00 & $\bar{z}$ & 19.19 \\
\hline gen & 55.11 & $\bar{z}$ & 91.52 & 57.17 & $\bar{z}$ & 93.13 \\
\hline gesa2 & 30.89 & $\bar{z}$ & 98.04 & 60.69 & $\bar{z}$ & 96.49 \\
\hline gesa2_o & 31.02 & $\bar{z}$ & 98.09 & 24.62 & $\bar{z}$ & 96.54 \\
\hline gesa3 & 45.76 & $\bar{z}$ & 62.99 & 65.28 & $\bar{z}$ & 72.24 \\
\hline gesa3_o & 49.16 & $\bar{z}$ & 69.98 & 60.06 & $\bar{z}$ & 71.03 \\
\hline markshare1 & 0.00 & $\geq$ & 0.00 & 0.00 & $\geq$ & 0.00 \\
\hline markshare2 & 0.00 & $\bar{z}$ & 0.00 & 0.00 & $\bar{z}$ & 0.00 \\
\hline mkc & 13.82 & $\bar{z}$ & 14.11 & 0.00 & $\bar{z}$ & 0.01 \\
\hline $\operatorname{misc} 03$ & 8.62 & $\bar{z}$ & 30.32 & 0.00 & $\bar{z}$ & 35.11 \\
\hline $\operatorname{misc} 07$ & 0.72 & $\bar{z}$ & 4.12 & 0.00 & $\bar{z}$ & 4.12 \\
\hline pp08a & 52.10 & $\bar{z}$ & 52.31 & 60.16 & $\bar{z}$ & 60.44 \\
\hline pp08aCUTS & 29.73 & $\bar{z}$ & 30.48 & 79.55 & $\bar{z}$ & 79.59 \\
\hline qiu & 0.27 & $\bar{z}$ & 7.85 & 0.00 & $\bar{\Sigma}$ & 10.71 \\
\hline qnet1 & 10.57 & $\bar{z}$ & 14.41 & 21.06 & $\bar{z}$ & 25.50 \\
\hline qnet1_o & 44.49 & $\bar{z}$ & 47.12 & 48.33 & $\bar{\Sigma}$ & 51.17 \\
\hline rout & 0.32 & $\bar{z}$ & 0.32 & 0.00 & $\bar{z}$ & 0.12 \\
\hline swath & 3.06 & $\bar{z}$ & 10.53 & 0.00 & $\bar{z}$ & 7.92 \\
\hline mas74 & 6.67 & & 6.67 & 4.14 & & 4.14 \\
\hline $\operatorname{mas} 76$ & 6.42 & & 6.42 & 5.15 & & 5.15 \\
\hline misc06 & 30.39 & & 30.39 & 0.00 & & 0.00 \\
\hline $\bmod 011$ & 1.67 & & 1.67 & 0.10 & & 0.10 \\
\hline modglob & 16.85 & & 16.85 & 13.22 & & 13.22 \\
\hline pk1 & 0.00 & & 0.00 & 0.00 & & 0.00 \\
\hline rgn & 1.61 & & 1.61 & 34.21 & & 34.21 \\
\hline
\end{tabular}

Table 2. Comparison with GMI cuts and MIR cuts

flugpl, where the pro-CG cuts improve greatly over all the other cuts combined. This indicates that the pro-CG cuts are genuinely different from those that are currently used in MILP solvers and that it is worth exploring heuristics that generate them more efficiently.

A second set of experiments has been performed to test the effectiveness of pro-CG cuts in the context of the simple model for the TW-ATSP discussed in Section 4, where the basic ILP model (22) only includes in- and out-degree 


\begin{tabular}{|c|c|c|c|c|c|c|c|c|}
\hline \multirow[b]{2}{*}{ instance } & \multicolumn{8}{|c|}{$\%$ gap closed } \\
\hline & & L\&P & & $\begin{array}{r}\mathrm{L} \& \mathrm{P} \\
+ \text { pro-CG } \\
\end{array}$ & & $\begin{array}{r}\text { GMI } \\
+\mathrm{MIR} \\
+\mathrm{L} \& \mathrm{P}\end{array}$ & & $\begin{array}{r}\text { GMI } \\
+\mathrm{MIR} \\
+\mathrm{L} \& \mathrm{P} \\
+ \text { pro-CG } \\
\end{array}$ \\
\hline bell3a & & 43.76 & & 81.47 & & 64.02 & & 91.68 \\
\hline bell5 & & 83.25 & & 92.82 & & 85.40 & & 93.18 \\
\hline egout & & 93.83 & & 98.84 & & 93.85 & & 100.00 \\
\hline fixnet6 & & 85.38 & & 91.96 & & 86.01 & & 92.33 \\
\hline khb05250 & & 99.39 & & 99.39 & & 98.43 & & 98.43 \\
\hline noswot & & - & & - & - & & - & \\
\hline rentacar & $\geq$ & 0.00 & & 0.00 & $\geq$ & 0.00 & & 0.00 \\
\hline set1ch & & 39.96 & & 68.88 & & 40.17 & & 69.27 \\
\hline vpm1 & & 31.40 & & 100.00 & & 53.90 & & 100.00 \\
\hline vpm2 & & 54.28 & & 79.05 & & 35.48 & & 69.22 \\
\hline 10teams & & 0.00 & $\geq$ & 57.14 & & 100.00 & & 100.00 \\
\hline arki001 & & 34.13 & $\geq$ & 34.13 & & 66.67 & $\geq$ & 79.19 \\
\hline blend 2 & & 21.56 & $\overline{\geq}$ & 35.86 & & 21.71 & $\bar{z}$ & 33.44 \\
\hline dano3mip & $\geq$ & 0.00 & $\geq$ & 0.00 & $\geq$ & 0.01 & $\geq$ & 0.01 \\
\hline danoint & $\bar{z}$ & 1.57 & $\bar{z}$ & 1.57 & $\bar{z}$ & 1.61 & $\bar{z}$ & 1.61 \\
\hline dcmulti & & 97.22 & $\geq$ & 97.30 & & 97.65 & $\geq$ & 97.95 \\
\hline dsbmip & & - & & - & & - & & 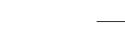 \\
\hline fiber & & 81.68 & $\geq$ & 83.30 & & 89.68 & $\geq$ & 91.39 \\
\hline flugpl & & 0.00 & $\overline{\geq}$ & 19.19 & & 11.74 & $\bar{z}$ & 41.75 \\
\hline gen & & 78.65 & $\geq$ & 92.29 & & 81.54 & $\geq$ & 97.05 \\
\hline gesa2 & & 37.83 & $\geq$ & 96.69 & & 81.55 & $\geq$ & 99.21 \\
\hline gesa2_o & & 37.83 & $\overline{\geq}$ & 98.60 & & 49.27 & $\bar{z}$ & 99.27 \\
\hline gesa3 & & 11.21 & $\geq$ & 58.30 & & 68.04 & $\geq$ & 71.05 \\
\hline gesa3_o & & 11.21 & $\overline{\geq}$ & 63.19 & & 68.12 & $\overline{\geq}$ & 74.79 \\
\hline markshare1 & & 0.00 & $\overline{\geq}$ & 0.00 & & 0.00 & $\bar{z}$ & 0.00 \\
\hline markshare2 & & 0.00 & $\overline{\geq}$ & 0.00 & & 0.00 & $\bar{z}$ & 0.00 \\
\hline $\mathrm{mkc}$ & $\geq$ & 26.82 & $\geq$ & 29.07 & $\geq$ & 36.65 & $\geq$ & 39.35 \\
\hline $\operatorname{misc} 03$ & & 39.67 & $\overline{\geq}$ & 44.91 & & 40.21 & $\bar{z}$ & 42.70 \\
\hline $\operatorname{misc} 07$ & & 12.03 & $\geq$ & 12.03 & & 12.25 & $\geq$ & 12.25 \\
\hline pp08a & & 80.46 & $\geq$ & 80.46 & & 81.35 & $\bar{z}$ & 81.35 \\
\hline pp08aCUTS & & 69.36 & $\overline{\geq}$ & 69.36 & & 88.87 & $\geq$ & 88.87 \\
\hline qiu & & 0.00 & $\bar{z}$ & 10.85 & & 28.79 & $\bar{z}$ & 28.95 \\
\hline qnet1 & & 6.61 & $\overline{\geq}$ & 11.99 & & 28.26 & $\geq$ & 31.54 \\
\hline qnet1_o & & 0.00 & $\overline{\geq}$ & 8.61 & & 48.39 & $\geq$ & 50.77 \\
\hline rout & & 30.09 & $\geq$ & 31.17 & & 30.51 & $\geq$ & 31.57 \\
\hline swath & $\geq$ & 0.32 & $\bar{z}$ & 8.41 & $\geq$ & 17.79 & $\bar{z}$ & 21.50 \\
\hline mas74 & & 0.00 & & 0.00 & & 6.84 & & 6.84 \\
\hline $\operatorname{mas} 76$ & & 0.00 & & 0.00 & & 7.03 & & 7.03 \\
\hline $\operatorname{misc} 06$ & & 79.52 & & 79.52 & & 46.51 & & 46.51 \\
\hline $\bmod 011$ & & 5.08 & & 5.08 & & 16.23 & & 16.23 \\
\hline modglob & & 57.08 & & 57.08 & & 60.76 & & 60.76 \\
\hline pk1 & & 0.00 & & 0.00 & & 0.00 & & 0.00 \\
\hline rgn & & 79.49 & & 79.49 & & 96.14 & & 96.14 \\
\hline
\end{tabular}

Table 3. Comparison with lift-and-project cuts and a combination of cuts. Note that for vpm2 and misc06, the gap closed by L\&P is larger than for GMI+MIR+L\&P. This happens because the L\&P cuts are strengthened and therefore there is no domination property 
equations (no subtour elimination constraints are exploited). Note that no continuous variables are present in the objective function of this model. Table 4 reports results on TW-ATSP real-world instances introduced by Ascheuer [1], derived "from an industry project with the aim to minimize the unloaded travel time of a stacker crane within an automated storage system".

\begin{tabular}{|c|c|c|c|c|c|c|c|c|}
\hline \multirow[b]{3}{*}{ instance } & \multirow[b]{3}{*}{$|I|$} & \multirow{3}{*}{$\begin{array}{r}\text { opt } \\
\text { value }\end{array}$} & \multicolumn{5}{|c|}{ pro-CG } & \multirow{3}{*}{$\begin{array}{r}{[2]} \\
\% \text { final } \\
\text { gap }\end{array}$} \\
\hline & & & & & \% gap & $\%$ time & $\%$ final & \\
\hline & & & \# iter.s & \# cuts & closed & to get the bound & gap & \\
\hline rbg010a & 12 & 149 & 227 & $526>$ & 99.07 & 7.50 & 0.67 & 0.67 \\
\hline $\operatorname{rbg} 017$ & 17 & 148 & 255 & $793 \geq$ & 78.07 & 27.89 & 14.86 & 0 \\
\hline rbg017.2 & 17 & 107 & 199 & $504 \geq$ & 96.90 & 27.80 & 1.87 & 0 \\
\hline rbg016a & 18 & 179 & 422 & $1,505 \geq$ & 97.08 & 100.00 & 1.68 & 1.11 \\
\hline rbg016b & 18 & 142 & 245 & $632 \geq$ & 86.54 & 31.77 & 10.56 & 6.33 \\
\hline rbg017a & 19 & 146 & 219 & $636 \geq$ & 95.07 & 39.42 & 2.74 & 0 \\
\hline rbg019a & 21 & 217 & 552 & $1,962 \geq$ & 97.40 & 100.00 & 1.38 & 0 \\
\hline rbg019b & 21 & 182 & 675 & $1,697 \geq$ & 89.47 & 100.00 & 6.59 & 1.09 \\
\hline $\operatorname{rbg} 019 c$ & 21 & 190 & 258 & $792 \geq$ & 70.21 & 23.12 & 20.53 & 4.21 \\
\hline rbg019d & 21 & 344 & 608 & $1,776 \geq$ & 90.57 & 100.00 & 4.94 & 0.29 \\
\hline $\operatorname{rbg} 021$ & 21 & 190 & 257 & $633 \geq$ & 72.05 & 20.27 & 20.53 & 4.21 \\
\hline $\operatorname{rbg} 021.2$ & 21 & 182 & 300 & $692 \geq$ & 77.00 & 25.32 & 17.03 & 0 \\
\hline $\operatorname{rbg} 021.3$ & 21 & 182 & 487 & $1348 \geq$ & 74.40 & 100.00 & 19.23 & 2.19 \\
\hline rbg021.4 & 21 & 179 & 416 & $1,134 \geq$ & 76.66 & 77.66 & 17.88 & 1.11 \\
\hline rbg021.5 & 21 & 169 & 306 & $908 \geq$ & 77.67 & 81.93 & 17.16 & 1.18 \\
\hline rbg021.6 & 21 & 134 & 294 & $743 \geq$ & 96.60 & 58.94 & 2.24 & 0.74 \\
\hline rbg021.7 & 21 & 133 & 263 & $658 \geq$ & 95.64 & 53.51 & 3.01 & 3.75 \\
\hline rbg021.8 & 21 & 132 & 346 & $744 \geq$ & 96.12 & 36.53 & 3.03 & 2.27 \\
\hline rbg021.9 & 21 & 132 & 369 & $761 \geq$ & 95.18 & 56.28 & 3.79 & 3.03 \\
\hline rbg020a & 22 & 210 & 399 & $1,150 \geq$ & 77.95 & 100.00 & 14.29 & 0 \\
\hline rbg027a & 29 & 268 & 667 & $1,655 \geq$ & 76.11 & 100.00 & 16.04 & 0.74 \\
\hline
\end{tabular}

Table 4. Stacker crane TW-ATSP instances

In particular, we report results on a set of 21 problems of small/medium size, with up to 30 vertices. The information provided in Table 4 for each instance is the number of cities $(|I|)$ and the optimal solution value (opt value). For pro-CG separation, Table 4 gives the same information as in Table 1 . As a comparison, we provide the final gap obtained by the pro-CG closure and the gap at the root node in [2] (note that we report the gap instead of the gap closed because a different initial formulation is used in [2]). Computing time is also not reported since the 1,200-second time limit is reached for all our TW-ATSP instances. Instead, we report the percentage of time (with respect to the time limit) spent to find the final bound. For example, for problem rbg016a the algorithm improves the bound from 42 to 148 in $90.1 \mathrm{CPU}$ seconds ( $7.50 \%$ of the total time) and spends the remaining computing time without finding any new cut. In such a case, we may guess that we are close to the Chvátal-Gomory closure, but proving that no violated pro-CG cut exists can require a great deal of enumeration.

The results for TW-ATSP instances are also very encouraging. Although our initial model is known to be very weak, pro-CG cuts are able to close a very significant amount (always more than 70\%) of the initial gap. This suggests that pro-CG cuts could be used successfully together with special purpose (polyhe- 
dral) separation routines in an attempt to improve the overall behavior of a cutting plane algorithm.

\section{Conclusions}

In this paper we have introduced a projected version of the classical CG cuts, and have studied their practical effectiveness for MIPLIB instances and for some special classes of MILP problems. Our approach is to project first the linear programming relaxation of the MILP at hand onto the space of the integer variables, and then to derive Chvátal-Gomory cuts for the projected polyhedron.

Although there are cases where they are ineffective, projected CG cuts provide excellent bounds for a number of MIPLIB instances. Furthermore, they can be applied successfully on a wide range of combinatorial problems where the continuous variables do not appear in the objective function. Our experiments on TW-ATSP confirm this claim-even starting from a very weak formulation involving big-M coefficients, the use of projected CG cuts is able to close a large portion of the integrality gap ( $70 \%$ or more, in our test cases). In our view, these results give a concrete hope that a similar performance can be obtained on other classes of problems (including scheduling and cutting/packing problems) when they are modeled through weak formulations involving continuous variables linked to the integer ones by constraints involving big-M coefficients.

\section{Acknowledgments}

We warmly thank Jon Lee and François Margot for friendly and useful discussions on the subject. Part of this research has been carried out when the last author was Herman Goldstine Fellow of the IBM T.J. Watson Research Center whose support is strongly acknowledged.

\section{References}

1. N. Ascheuer, Hamiltonian Path Problems in the On-line Optimization of Flexible Manufacturing Systems, PhD thesis, Technische Universität Berlin, Berlin, Germany, 1995.

2. N. Ascheuer, M. Fischetti, M. Grötschel, A polyhedral study of the asymmetric travelling salesman problem with time windows, Networks 36, 69-79, 2000.

3. E. Balas, S. Ceria and G. Cornuéjols, A lift-and-project cutting plane algorithm for mixed 0-1 programs, Mathematical Programming 58 (1993) 295-324.

4. E. Balas, A. Saxena, Optimizing over the split closure: Modeling and theoretical analysis, IMA "Hot Topics" Workshop: Mixed Integer Programming, Minneapolis, July 25-29 2005.

5. R.E. Bixby, S. Ceria, C.M. McZeal, M.W.P. Savelsbergh, MIPLIB 3.0, http://www. caam.rice.edu/ bixby/miplib/miplib.html.

6. P. Bonami and M. Minoux, Using rank-1 lift-and-project closures to generate cuts for 0-1 MIPs, a computational investigation, Discrete Optimization 2, 288-307, 2005.

7. A. Caprara, A.N. Letchford, On the separation of split cuts and related inequalities, Mathematical Programming 94, 279-294, 2003.

8. V. Chvátal, Edmonds polytopes and a hierarchy of combinatorial problems, Discrete Mathematics 4, 305-337, 1973. 
9. G. Codato, M. Fischetti, Combinatorial Benders' cuts, Operations Research, to appear, 2005.

10. COIN-OR. www.coin-or.org

11. W. Cook, R. Kannan, A. Schrijver, Chvátal closures for mixed integer programming problems, Mathematical Programming 47, 155-174, 1990.

12. G. Cornuéjols, Y. Li, On the rank of mixed 0,1 polyhedra, Mathematical Programming 91, 391-397, 2002.

13. G. Cornuéjols, Y. Li, A connection between cutting plane theory and the geometry of numbers, Mathematical Programming 93, 123-127, 2002.

14. S. Dash, O. Günlük, A. Lodi, On the MIR closure of polyhedra. IBM, T.J. Watson Research, Working paper, 2005.

15. F. Eisenbrand, On the membership problem for the elementary closure of a polyhedron, Combinatorica 19, 297-300, 1999.

16. M. Fischetti, A. Lodi, Optimizing over the first Chvátal closure, in M. Jünger and V. Kaibel (ed.s), Integer Programming and Combinatorial Optimization - IPCO 2005, LNCS 3509, Springer-Verlag, Berlin Heidelberg, 12-22, 2005.

17. R.E. Gomory, Outline of an algorithm for integer solutions to linear programs, Bulletin of the AMS 64, 275-278, 1958 .

18. R.E. Gomory, An algorithm for integer solutions to linear programs, in R.L. Graves and P. Wolfe (eds) Recent Advances in Mathematical Programming, McGraw-Hill, New York, 269-302, 1963.

19. M. Grötschel, L. Lovász, A. Schrijver, Geometric Algorithms and Combinatorial Optimization, Springer-Verlag, Berlin, 1988.

20. ILOG Cplex 9.0: User's Manual and Reference Manual, ILOG, S.A., http://www.ilog.com/, 2005.

21. G. W. Klau, P. Mützel, Optimal labelling of point features in rectangular labelling models, Mathematical Programming 94, 435-458, 2003.

22. H. Marchand and L.A. Wolsey, Aggregation and mixed integer rounding to solve MIPs, Operations Research 49, 363-371, 2001.

23. G.L. Nemhauser, L.A. Wolsey, Integer and Combinatorial Optimization, Wiley, New York, 1988.

24. G.L. Nemhauser, L.A. Wolsey, A recursive procedure to generate all cuts for 0-1 mixed integer programs, Mathematical Programming 46, 379-390, 1990.

25. A. Schrijver, On cutting planes, Annals of Discrete Mathematics 9, 291-296, 1980. 